\title{
Iterative Multipacket Detection with FDE based MAC Protocol in Vehicular Ad Hoc Networks by Belal Al-khateeb
}

Submission date: 06-Aug-2019 12:51AM (UTC+0400)

Submission ID: 1157895440

File name: OSA3.docx (824.63K)

Word count: 5245

Character count: 29001 


\title{
Iterative Multipacket Detection with FDE based MAC Protocol in Vehicular Ad Hoc Networks
}

\author{
Salah A. Aliesawi ${ }^{1}$, Mohammed Sh. Ahmed ${ }^{2}$, Ahmed N. Rashid ${ }^{3}$ \\ ${ }^{1,3}$ Department of Computer Science/College of Computer Science and Information Technology, University of Anbar \\ ${ }^{2}$ College of Petroleum and Minerals Engineering, University of Tikrit
}

\begin{tabular}{ll}
\hline Article Info & ABSTRACT \\
\hline Received & Wireless access in vehicular environments (WAVE), is especially designed to \\
support vehicular ad hoc networks (VANETs) requirements, where rapidly \\
changing channel conditions introduces unsynchronized transmissions. In such \\
networks, instead of dealing with interferences in medium access control \\
(MAC) layer or physical layer alone, both layers should be considered to \\
cooperate and complement each other. In this paper, multipacket detection \\
(MPD) technique with frequency domain equalization (FDE) is proposed for \\
Keyword: & VANETs, with cyclically shifted different interleavers for different nodes, to \\
remove interference and reducing the information exchange between nodes. \\
Theless networks \\
These promising multi-hop wireless networks are used in situations, where \\
temporary network connectivity is needed. Therefore, to improve the \\
Interleavers \\
communication between vehicles (V2V) and from vehicles to roadside \\
infrastructure (V2I), MPD-FDE with interference cancellation (IC) schemes \\
can be used iteratively to successfully decode and receive even colliding \\
packets. For designing such a protocol, different key aspects are discussed with \\
an emphasis on iterative MPD-FDE. Numerical results with different network \\
nodes, show the MPD-FDE performances for coded and uncoded transmissions \\
with different IC schemes, where successive IC (SIC) is much better than \\
parallel IC (PIC) schemes. It is also shown that the proposed protocol provide \\
reliable detection and excellent throughput improvements, with much less \\
resource consumption compared to multiple random interleavers.
\end{tabular}

\section{Corresponding Author:}

Salah A. Aliesawi,

Computer Science Departement,

College of Computer Science and Information Technology, University of Anbar

Email: salaheng1996@gmail.com

\section{Introduction}

Vehicular ad-hoc networks (VANETs), are one of the important components that needed to develop intelligent transportation systems (ITS). In ITS, the wireless access is made between vehicles and between the vehicles and roadside unit (RSU). These two distinct types of net 3 r rking, vehicle-to-vehicle (V2V) and vehicle-toinfrastructure (V2I), have enabled a variety of applications in decreasing the accidents and traffic congestions. The V2I networking is used to exchange information with RSU, when the vehicles within the transmission range. By periodically exchanging up-to-date traffic information with neighboring vehicles, e.g., driving speed, position and heading, traffic safety applications are envisioned to detect dangerous traffic situations in advance and sending warning messages. Furthermore, vehicular networks offer a wide 3 nge of applications such as increasing efficiency of the public transport systems, decreasing the travelling time and saving fuel that has large economic benefits [1],[2]. 
In vehicular networks, IEEE 802.11p defines a medium access control (MAC) and a physical (PHY) layer for WAVE. IEEE 802.11p exchanges the messages, Request to Send (RTS) and Clear to Send (CTS), to avoid the timely overlap of two or more packets at a receiver [3]. However, the rapidly changing topology and channel conditions, due to the high mobility of moving vehicles, introduce unsynchronized transmissions, which have a significant negative impact on the effectiveness of this technique. In such systems, the introduced overhead is not acceptable, considering that the contact duration times will be rather small. Further, the close surrounding nodes might not detect an ongoing transmission and interfere when they actually should not [1],[4],[5]. Therefore, MAC and PHY layer functioning in high mobility environments, have to be developed using recent advances in multiuser communications [6],[7].

In this paper, multipacket detection (MPD) scheme with frequency domain equalization (FDE), is proposed with node-specific interleaving to reduce interference, thereby improving the spatial utilization of wireless medium. Unfortunately, it is not direct to use such systems in ad hoc networks, where traditional MAC protocols see the PHY layer as a collision model and attempts to prevent simultaneous communications (e.g. [8]). This paper is structured as: section 2 focuses on the related works, while the VANETs architecture is introduced in section 3, with brief description of the traditional MAC protocols. Section 4 describes the mathematical formulations of MPD-FDE with briefly system model, followed by the key needs for developing an integrated MAC protocol in front of the physical layer. The simulation results will be shown in section 5 . Finally, conclusions and future directions are made in section 6.

\section{Related Works : MAC and PHY Layer}

In IEEE 802.11 standard, MAC protocols are generally designed to blocks all neighboring nodes and avoid simultaneous transmissions, by exchanging RTS and CTS messages. Therefore, it has low efficiency due to the such blocking mechanism. From information theory, it is possible to successfully decode even overlapping/ colliding packets and improve the spatial utilization, mostly by using multiple antennas [9],[10], and interference cancellation (IC) algorithms, which are necessary to improve the detection process in PHY layer. In $[11,12]$ a successive IC (SIC) algorithm is used to address the interference problem, and it has already been applied to ZigBee [13]. In SIC algorithm, the receiving node detects the bits of the stronger frame. Then, the interference produced by the stronger frame is removed from the signal to obtain a clean signal that consists only the noise and the bits of the weaker frame. In multi overlapping frames, the process is repeated to detect all the frames. By contrast, in parallel IC (PIC) scheme [14],[15], the bits of different frames/users are detected and the interference caused by other users/frames is removed simultaneously. Both IC algorithms have problems of error propagation that results more errors in next detection. Further, the SIC has long time delay with large number of users. In [2], the ordered SIC (OSIC) scheme with increased complexity is used to address the problem and get better performance from prioritizing the users. In IEEE $802.11 \mathrm{p}$, MAC protocols use simple ALOHA, and orthogonal frequency-division multiplexing (OFDM) technology is used as a physical layer, to increase data rate and overcome signal fading. However, the decoding process with many nodes becomes highly complex, and the solution becomes unsatisfactory [16]. Turbo techniques that exchange soft information between two soft input soft output (SISO) units, are used in [17], to improve the performance in such systems. In [4], the researchers emphasized that the MAC and PHY layers, should be develop to work and complement each other [4],[5].

On the other hand, spread spectrum system (SSS) based PHY layer is used in IEEE 802.11 [18]. In [19], code division multiple access (CDMA) systems have shown great enhancement in detection and relieve the near-far problem. The possible solution to cope with such problem is used multiuser detectors (MUDs) at physical layer, e.g. , [7], [17]. The existing MUD works are often considered to encourage simultaneous transmission. In such networks, to make SSS competitive, synchronization and signature exchange need to be also addressed as in [4],[20]. To date, all these studies in VANETs, have been in the time domain, such as CDMA and interleave division multiple access (IDMA6 However, interference can be resolved ef 6 ciently using turbo FD techniques. In contrast to time domain, the related detection costs are very low and it is independent of simultaneous user 6 imber and path number. Further, single carrier systems with FDE, have similar performance and efficiency as orthogonal frequency division multiplexing (OFDM), while alleviating the peak to average transmitted power and carrier synchronization difficulties in OFDM [21]-[23]. 


\section{3. Vehicular Ad Hoc Networks: Architecture and Access Technology}

In recent years, VANETs have improved transportation safety, by combining with existing technology, such as satellite, wireless communication, and sensing. In Fig. 1, wireless network uses wireless communication between vehicles on the road, for example bus, car, and truck. It is considered as peer to peer, multi hop wireless networks, where communication is forwarded from source vehicle to destination vehicle in hop to hop basis. In VANET, vehicles are restricted to specific paths and directions [34-37].

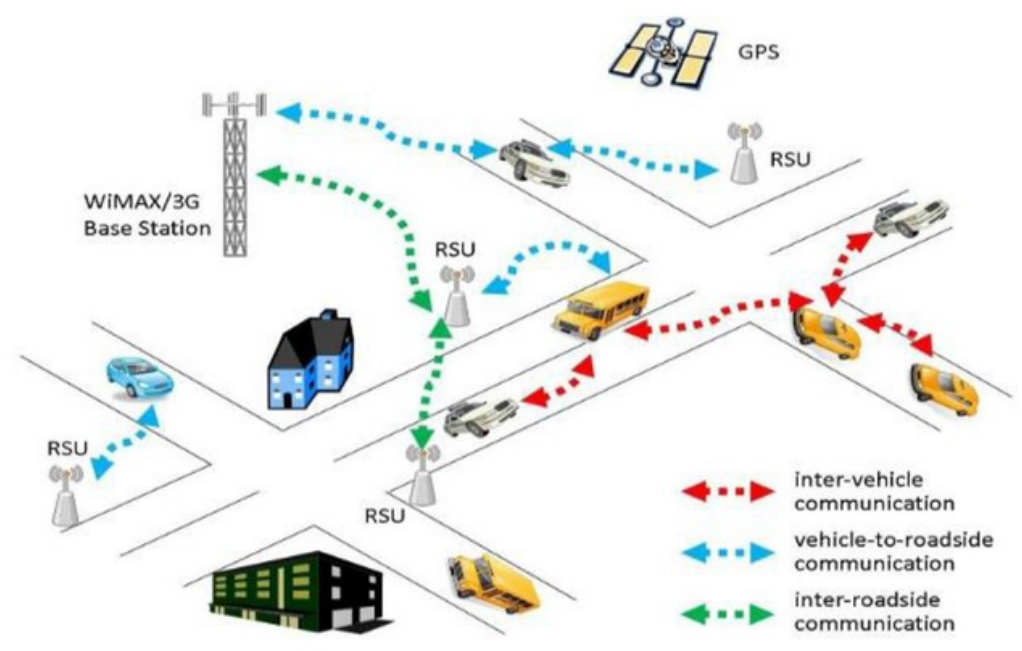

Fig. (1) Illustration of VANETs [24]

Therefore, it can be supported by RSUs that can provide safety services for cars. The safety-related applications that build an ITS, represent the main aim of inter-vehicular communication. These applications can collect information from RSUs or surrounding vehicles, analyses 5 he information and notify other vehicles. When an accident is detected or a car brakes suddenly, these safety applications broadcast messages to neighboring cars and warns them 5 bout this situation. However, vehicles do not expect to receive any response and need to follow same standards to be able to exchange information [24]-[29].

\subsection{Network Components and Communication Types}

5

Vehicles are performed as mobile nodes and equipped with dedicated short range communication (DSRC) technolo5. In such networks, the two types of wireless access can be used with RSUs and on-board units (OBUs), which are fixed on a vehicle and can operate with moving. In rural areas, RSUs are not widely deployed due to the high costs. These units host peer applications and implement the communication protocol and algorithms. There are also some applications on devices to provide services for OBUs. Fig. 2 highlights the types of communications in such networks, where vehicles can communicate with other vehicles, i.e. V2V, but also communication from a vehicle to the infrastructure (V2I) is possible, or vice versa. Every vehicle tries to look for an infrastructure within the communication range to establish desired connection and understanding of their environment. Vehicle $\mathrm{A}$ and $\mathrm{B}$ are within the communication range, whereas vehicle $\mathrm{G}$ and D are communicating with RSUs [30]. 


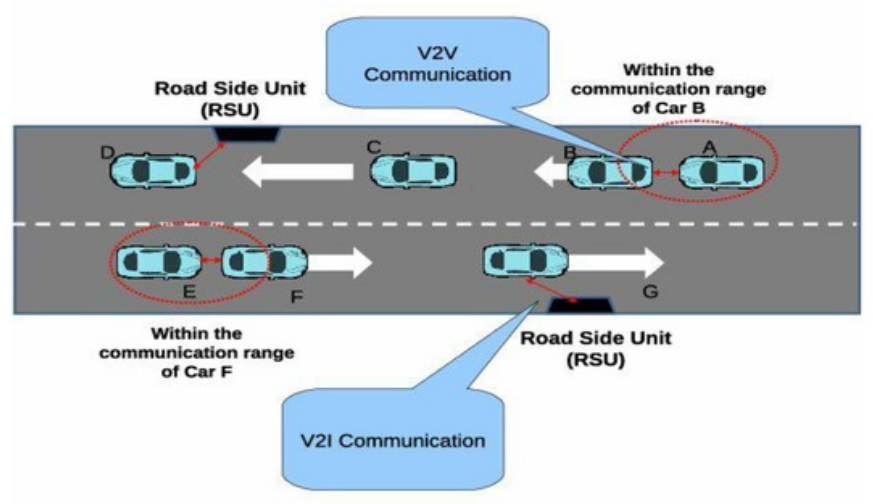

Fig. (2) Different types of communication

\subsection{Layered Architecture for VANETs}

The standardization of a ${ }_{4}$ hitectures is needed to ensure co-operability between different brands and different countries. It may ${ }_{4}$ hange from region to region, thus, the protocols and interfaces are also different among them. In the US, the dedicated short range communication (DSRC) with protocol stack in Fig 4 , is specifically designed to provide services and interfaces for V2V and V2I wireless communications. Wireless access in vehicular environments (WAVE) is most often used together with the DSRC. It is a suite of standards that are concentrated on the wireless bands and technologies [31]. However, different protocols are specially designed to support VANETs requirements.

\begin{tabular}{|cc|}
\hline $\begin{array}{c}\text { Safety } \\
\text { applications }\end{array}$ & $\begin{array}{c}\text { Nonsafety } \\
\text { applications }\end{array}$ \\
\hline $\begin{array}{c}\text { Transport and } \\
\text { network layer } \\
\text { IEEE } 1609.3 \\
\text { Security } \\
\text { IEEE 1609.2 }\end{array}$ & $\begin{array}{c}\text { Transport layer } \\
\text { TCP/UDP } \\
\text { Network layer } \\
\text { IPv6 }\end{array}$ \\
\hline & LLC sublayer \\
IFEE 802.2 & \\
\hline & MAC sublayer extension \\
\hline IEFE 1609.4 \\
\hline MAC sublayer \\
PHY layer
\end{tabular}

Fig. (3) DSRC communication's layered architecture [31]

The applications layer present the ITS applications using the lower layers to connect to other ITS applica 4 ions. Two or more connected ITS applications together form an application providing an ITS service to a user. IEEE 
409 characterizes a family of standards that support safety applications for WAVE. In non-safety applications, network layer and transport layer services are delivered using set of protocols, such as UDP, TCP, and IPv6 [31],[32]. IEEE 802.11p specifies the set of protocols in the physical (PHY) and MAC layers for implementing WAVE. The data link layer (DLL) is subdivided into MAC and Link Layer Control (LLC). PHY layer connects physically to the communication medium and a data link layer for end-to-end data transfer. It supports $10 \mathrm{MHz}$ bandwidth and takes care of error correction codes, modulation/demodulation, etc. The MAC layer is an important sub-layer of the DLL, and it defines interface functions and signaling techniques. It takes care of messages, when a station is allowed to access the shared medium to reduce collisions. The LLC is used for multiplexing protocols transmitted over the MAC layer. In the next subsections the PHY and MAC of DSRC, based on IEEE 802.11 p, are discussed. In the next section, the proposed protocol is presented and designed to satisfy the important requirements for multiuser detection aware MAC protocols.

\subsection{Traditional MAC Protocols}

MAC protocols can be classified as being either contention based, for examples Aloha, carrier sense multiple access (CSMA) and CSMA with collision avoidance (CSMA/CA), or contention-free protocols, for examples time division multiple access (TDMA), and frequency division multiple access (FDMA). In Aloha protocol, a station can access the medium at any time for data transmission and it does not impose any overhead due to access mechanisms. In CSMA, the carrier is sensed before it is accessed and it is preferred when the loads get higher. Aloha and CSMA are not able to meet real-time requirements [33]. The CSMA/CA adds a back-off time within a certain contention window $(\mathrm{CW})$, in case of a busy medium to achieve fairness among competing stations, and to decrease the probability of a collision. In contention-free protocols, stations are assigned time slots respectively to transmit data. However, the drawback of these protocols is the need for a central allocation mechanism [27].

\section{MAC and Multipacket Detection (MPD) Protocol}

\subsection{MAC-MPD: Frame Structure and Protocol Description}

In MAC-MPD, data packets are organized in successive blocks, $b \in\{1, \ldots, B\}$, of equal duration. These blocks are continuously repeated over time as shown in Fig. 4. The transmitted frame employed payload data and slots for announcements (ann.), objections (obj.), acknowledgment (ack.) messages, separated by gap periods to avoid interpacket interference [4]. The transmitted packets have to be broadcasted by transmitter using ann slot, and simultaneous receivers could object the transmission using obj slot, depending on their detection capabilities. Simultaneous transmissions of such signaling messages regarded as collisions. Therefore, each node starts transmitting with " mini - slots" or random delay, unless it detects any ann messages from other nodes. "Mini - slots" are used to reduce the probability of ann collisions. A collision happens every time two or more nodes choose to transmit in the same "mini-slots". How 2 er, MPD-FDE is not applied during (ann , obj , ack) slots, and it is used with the slot of data block. Thus, the detection of such messages does not require strong processing capabilities.

By using the corresponding ann slot, the receiving node listens for ann messages. If ann is not detected, the receiving node does need to send an obj message. Otherwise, the receiving node will extract the interleaver/signature information and removing out the interference using MPD-FDE. If MPD-FDE is not able to deal with the expected interference, the receiving node will subsequently send an $o b j$ message using $o b j$ slot. If no objection occurs, the transmitter will continue with the packet transmission, otherwise it will make another attempt at later time. Further, MPD-FDE will not able to remove the interference, if the interleaver sequence cannot be extracted from ann messages. 
Communication Sessions

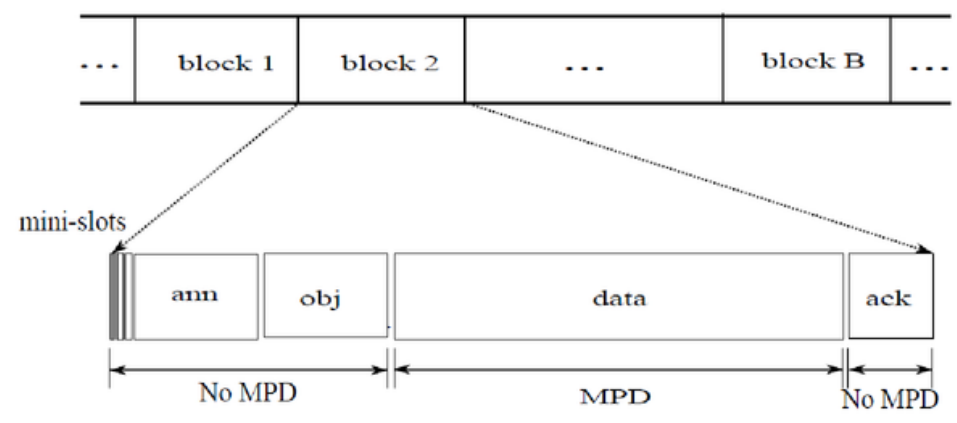

Fig. (4) Frame structure of MAC-MPD protocol

$I_{2}$ the data is successfully decoded, the receiver will send ack message per packet. In compared to the traditional MAC protocol, where both RTS and CTS messages are used to block neighboring nodes, only receiving nodes can avoid a transmission demand or ann messages. Therefore, no exposed terminal problem in MAC-MPD is caused as in IEEE $802.11 \mathrm{p}$.

\subsection{Information Exchange of Multiple Interleavers}

In general, spread spectrum systems (SSSs) support simultaneous transmissions and separate signals using userspecific interleavers/spreading codes. In ad hoc networks, these user-specific interleavers have to be allocated to different nodes and updated according to topology changes without a base station. Therefore, it is hard to manage multiple interleavers among multiple nodes. Further, receiving nodes must monitor the channel to determine which and when nodes begin transmitting their data to remove incoming interference [20].

In ad hoc networks, each node with MPD-FDE, requires to know the node-specific interleaver $\left(\Pi_{n}\right)$ and all interleavers of neighboring nodes. The information of these interleavers has to be exchanged as a part of ann messages. In practical systems, to reduce signaling overhead and memory requirements, a master interleaver (П) with cyclic shift can be used to generate multiple interleavers as depicted in Fig. 5.

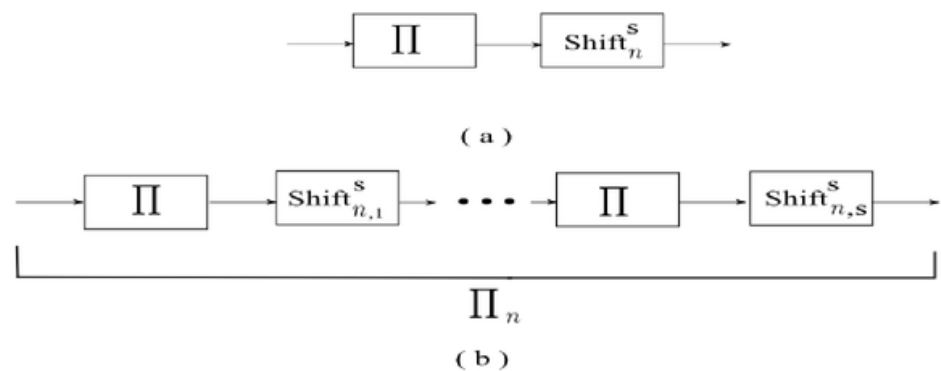

Fig. ( 5 ) Generation steps of cyclically shifted interleavers: ( a ) master interleaver with cyclic shift, (b ) repeat (a) with multiple cyclic shifts. 
For the node $n$, node-specific interleaver $\left(\Pi_{n}\right)$ is generated by circular shifting with $s$ steps of the master interleaver ( $\Pi$ ) as

$$
\Pi_{n}=F_{f}(\Pi, s)
$$

where $f$ denotes an update frequency of the $N$ multiple interleavers to decrease the probability of collisions. Each transceiver only exchanges the seed or parameters $(\Pi, s, f)$, which is used efficiently to generate multiple interleavers if the desired node knows the step shifting for each node. The master interleaver $\Pi$ and cyclic shifts $s$ are randomly generated from a uniform distribution. Further, $s=1$ or 2 , will be inadequate for a good node separation and high errors can be observed.

\subsection{PHY layer: Data Transmission Process}

We consider a V2R communication with multiple nodes, $1 \leq n \leq N$, communicating simultaneously with a single RSU, as shown in Fig. 6. The RSU and each node are configured with a single antenna. All nodes transmit to RSU in a broadcast manner and the channels are assumed to be a typical highway vehicular scenario. It consists of a RSU acting as a receiver (Rx), and a vehicle acting as a transmitter (Tx). Since we want to simulate a V2R scenario, the Rx does not move and could be located in the middle of the road. Fig. 7 (a) shows the block structure of the PHY transmission process, where only a node with MPD-FDE can receive connections simultaneously.

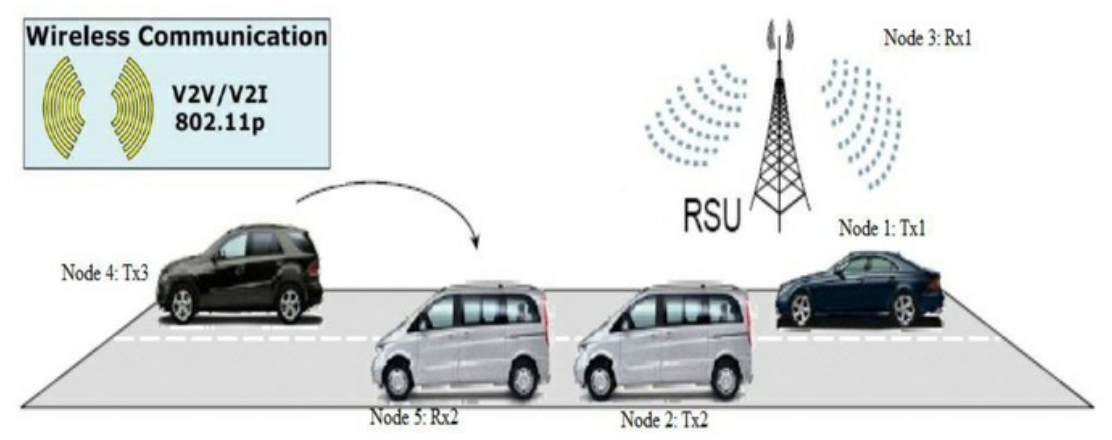

Fig. (6) Two vehicles (nodes 1, 2), single RSU (node 3) and interferer (node 4) scenario

\subsubsection{Transmitter Side}

The transmitter (Tx) structure, with channel coding and node-specific interleaver, is shown in Fig.7(b). Information bits are first encoded using convolutional code (CC) with rate $R$, and spreaded using a length-sp repetition code $(\mathrm{RC}),[-1,1,-1, \ldots,-1,1]$. In IEEE $802.11 \mathrm{p}, \mathrm{CC}$ uses $R$ with $\left(\frac{1}{2}, \frac{3}{4}\right.$ or $\left.\frac{2}{3}\right)$ to correct the errors in received signals. The coded bits are independently permuted by a node-specific interleaver for the $n$th node, $\left(\Pi_{n}\right)$. Then, they are mapped onto the complex symbols using QPSK signal mapper. The resulting transmitted signal, $\left\{x_{n, m}\right\}_{m}^{M}$, is multiplexed with cyclic prefix (CP) of length $L$. CP can avoid interblock interference by converting linear convolution to cyclic convolution. Thus, the received signal $r_{n}$ of the $n$th node, after transmitting over wireless channels can be written as

$$
r_{n}=\sum_{n=1}^{N} \quad H_{n} x_{n}+w
$$




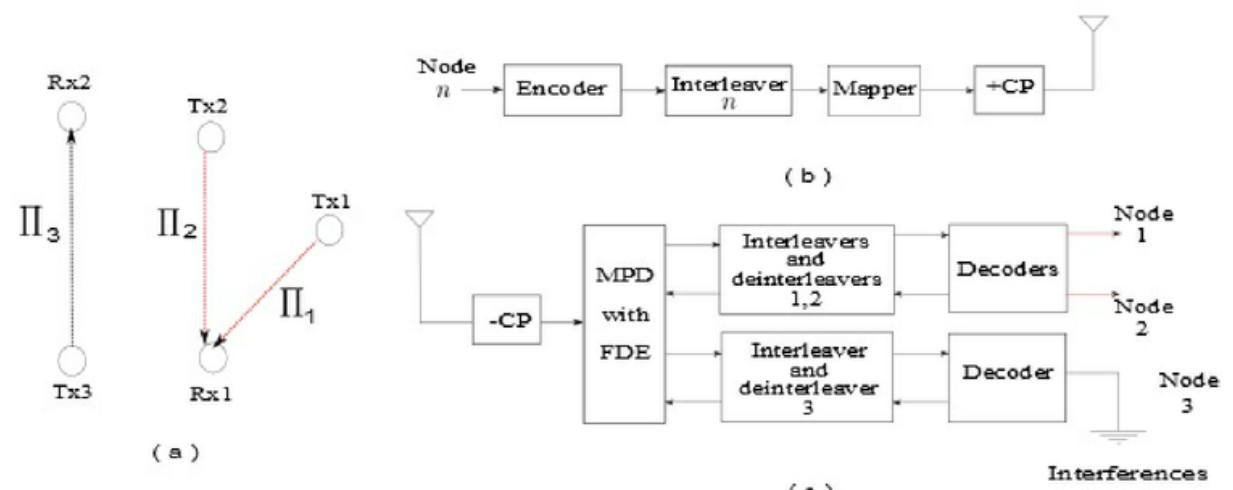

(c)

Fig. (7) Block diagrams of PHY data transmissions process, (a) transmission scenario (b) transmitter ( Tx ) structure ( c ) MPD-FDE Receiver (Rx) as a means of simultaneous transmissions and IC in VANETs.

where $x_{n}=\left[\begin{array}{llll}x_{n, 0} & x_{n, 1} \ldots x_{n, M-1}\end{array}\right]^{T}$, represents the transmitted vector of the $n$th node. $\mathrm{w}$ is the additive white noise vector with zero mean and covariance matrix $o_{w}^{2} I . H_{n}$ is a circulated matrix with the first column, $h=$ $\left[h_{n, 0} h_{n, 1} \ldots h_{n, L} 0 \ldots 0\right]^{T}$.

\subsubsection{Multipacket Detection (MPD) with FDE}

The simplified block diagram of the MPD with FDE is shown in Fig. 7(c). The inserted CP must be removed to obtain the transmitted data. The received vector, $z_{n}=\left[z_{n, 0} z_{n, 1} \ldots z_{n, M-1}\right]$ of the $n$th node, after removing remaining interferers from $r$ using MPD, can be written as [21]

$$
z_{n}=H_{n} x_{n}+\sum_{n^{\prime}=1, n \neq n}^{N} \quad H_{n^{\prime}}\left(x_{n^{\prime}}-\underline{x}_{n^{\prime}}\right)+w,
$$

where $\underline{x}_{n}=\left[\underline{x}_{n, 0} \underline{x}_{n, 1} \ldots \underline{x}_{n, N-1}\right]^{T}$, represents the mean vector of the transmitted symbols $\left\{x_{n, m}\right\}_{m=0}^{M-1}$ from the decoder of the $n$th node. It is obtained for each QPSK symbol using the feedback information from the decoders in the previous iteration, $\left\{L_{B A}\left(c_{n, m}\right)\right\}_{m=0}^{2 M-1}=\Pi_{n}\left(\left\{L_{D}\left(c_{n, l}\right)\right\}_{l=0}^{2 M-1}\right)$, as

$$
\underline{x}_{n, m}=\tanh \tanh \left(\frac{L_{B A}\left(c_{n, 2 m}\right)}{2}\right)+i \tanh \tanh \left(\frac{L_{B A}\left(c_{n, 2 m+1}\right)}{2}\right)
$$

The decoders compute the extrinsic information for both the coded, $\left\{L_{D A}\left(c_{n, l}\right)\right\}_{l=0}^{2 M-1}=\Pi_{n}^{-1}\left(\left\{L_{B}\left(c_{n, m}\right)\right\}_{m=0}^{2 M-1}\right)$, and decoded bits. These soft values are used as a priori information of the MPD-FDE unit. The frequency domain (FD) estimates, $\left\{\hat{X}_{n, m}\right\}_{m=0}^{M-1}$, of $\left\{x_{n, m}\right\}_{m=0}^{M-1}$, can be computed from the received symbols, $\left\{r_{n}\right\}_{m=0}^{M-1}$ and the a priori information, $\left\{L_{B A}\left(c_{n, m}\right)\right\}_{m=0}^{2 M-1}$, by minimizing the mean-square error (MSE), $E\left\{\left|x_{n, m}-\hat{x}_{n, m}\right|^{2}\right\}$. The reconstructed symbols, $\left\{\hat{x}_{n, m}\right\}_{m=0}^{M-1}$, are calculated as

$$
\hat{x}_{n, m}=E\left\{x_{n, m}\right\}+g_{n}^{H}\left(z_{n}-E\left\{z_{n}\right\}\right), 0 \leq m<M,
$$

where $g_{n}$ denotes the weight vector of the equalization, and $(.)^{H}$ is the conjugate transpose. The extrinsic LLRs of MPD, $\left.L_{B}\left(c_{n, m}\right)\right\}_{m=0}^{2 M-1}$ for each symbol, are obtained as

$$
L_{B}\left(c_{n, 2 m}\right)=\frac{2 \sqrt{2} \operatorname{Re}\left\{\hat{x}_{n, m}\right\} \mu_{n}}{\sigma_{n}^{2}}
$$




$$
L_{B}\left(c_{n, 2 m+1}\right)=\frac{2 \sqrt{2} \operatorname{Im}\left\{\hat{x}_{n, m}\right\} \mu_{n}}{\sigma_{n}^{2}}
$$

where $\mu_{n}=\frac{1}{N} \sum_{k=0}^{N-1} \quad\left(G_{n, k}^{*} H_{n, k}\right)$, and $\sigma_{n}^{2}=\mu_{n}-v_{n} \mu_{n}^{2}$, are the mean and variance of the estimates $\left\{\hat{x}_{n, m}\right\}_{m=0}^{M-1}$, with $G_{n, k}=\frac{H_{u, k}}{\sigma_{w}^{2}+\sum_{n=1}^{N} v_{n}\left|H_{n, k}\right|^{2}}$, and a diagonal matrix $H_{n}=F H_{n} F^{-1}$, respectively. $F=$ [ $\left.\begin{array}{llll}1 & 1 & \cdots & 1\end{array}\right]^{T}$ and $H_{n, k}$ with $(k, k)$ entry equals to the $k t$ hFT coefficient of the channel impulse response for the nth node [23]. The iterative procedure can be described briefly as

Step 1: compute the means, $\mu_{n}$ and variances, $\sigma_{n}^{2}$, using the feedback information from the decoders. For iteration $(i t)=1, \mu_{n}$ and $\sigma_{n}^{2}$ are zero and one, respectively.

Step 2: obtain the equalized symbols, $\hat{x}_{n, m}$ using the equalization process in (5).

Step 3: compute the extrinsic estimates, $L_{B}\left(c_{n, 2 m}\right)$, using (6), and feed them after deinterleaving, $\Pi_{n}^{-1}$, to the decoders. The decoder computes the $L_{B A}\left(c_{u, l}\right)$ of the coded bits, and these bits are used as a priori information of MPD-FDE unit.

\section{Numerical Results and Discussion}

\subsection{Simulation Scenarios and Parameters}

For the uncoded transmission, simulation parameters are illustrated as follows: 250 frames with 1024 bits are encoded only by repetition code with $s p=16$. These frames are transmitted using Tx in Fig. 7 (b) with the scenario shown in Fig. 7 (a). The interleaved chips are modulated using QPSK symbols, and then transmitted over vehicular channels. The channels are assumed to be line-of-sight with multi-paths fading coefficients. The nature of the V2V and V2I communication, due to the multi fading and scatting environments, adds more challenges to estimate the channel characteristics. The repetition codes are common for all nodes with constant power and yields alternating bits. The cyclic prefix $(C P)$ with 20 symbols is employed to reduce the interpacket interference. The receiving node applies the MPD-FDE as shown in Fig. 7 (c) and explained in Section 4.3. In coded system, a binary convolutional encoder with $R=1 / 2$ is used with $g=[23 ; 35]_{8}$. A topology with $N=2$ to 24 communication pairs are studied and each communication pair is assumed to be within a distance of less than $100 \mathrm{~m}$. The PHY layer performance of the standard IEEE $802.11 \mathrm{p}$, can be used as a reference for the whole vehicular wireless network.

\subsection{Performance with different iterations and block sizes}

Fig. 8 shows the performance of $\mathrm{M} 2$ )-FDE with SIC scheme for different block sizes $\left(N_{b}\right)$ and iterations (it ). BER is averaged over $N=4$ nodes and the result is found after 4 iterations. For large block sizes $\left(N_{b}\right)$, larger interleaver is guaranteed because bandwidth expansion is exploited before the interleaver, which is favorable for iterative processing. In contrast, the performance degradations is high for short block sizes. In first iteration, the BER is worse due to high noise that results more errors. However, error propagation becomes under control with high SNR regions. Thus, the SIC scheme shows its iterative detection gain.

\subsection{Effects of interleavers and number of nodes}

In this section, numerical results of the proposed detector with (it $=4, s p=16)$ are drawn in Fig. 9, for different $N$ and interleavers. For comparison, PHY layer performance of standard IEEE 802.1 lp also plotted as a single user bound. In Fig. 9 ( b), the BER of the proposed system with randomly generated interleavers is compared with the case of using cyclically shifted interleavers. It can be observed that the BER of the cyclically shifted interleavers close to that of randomly generated interleavers. Since the interleavers have a large portion of small interfering weights which degrade the error performance. However, the memory requirement of the random interleavers is $(8 \times 1024 \times 16)$ bits for 8 nodes and $(24 \times 1024 \times 16)$ bits for 24 nodes, whereas the proposed interleavers need $(1 \times 1024 \times 16+210)$ for the nodes $=8$ and 24 . Thus, cyclically shifted interleavers are much convenient than the randomly interleavers for ad hoc networks. Further, they are independent on the number of 
nodes, and can reduce the information exchange between $\mathrm{Tx}$ and $\mathrm{Rx}$. In practical systems, choosing dense netwo $2 \mathrm{k}$ topologies with larger nodes, may cause significant performance degradation as in Fig. 9 (a). Thus, the BER degradation becomes severe as the interference level goes up with $N=24$.

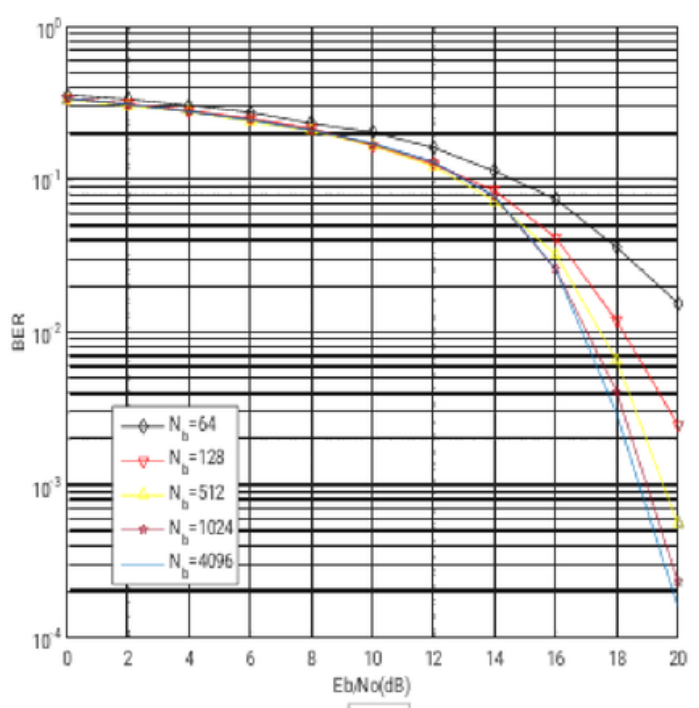

(a)

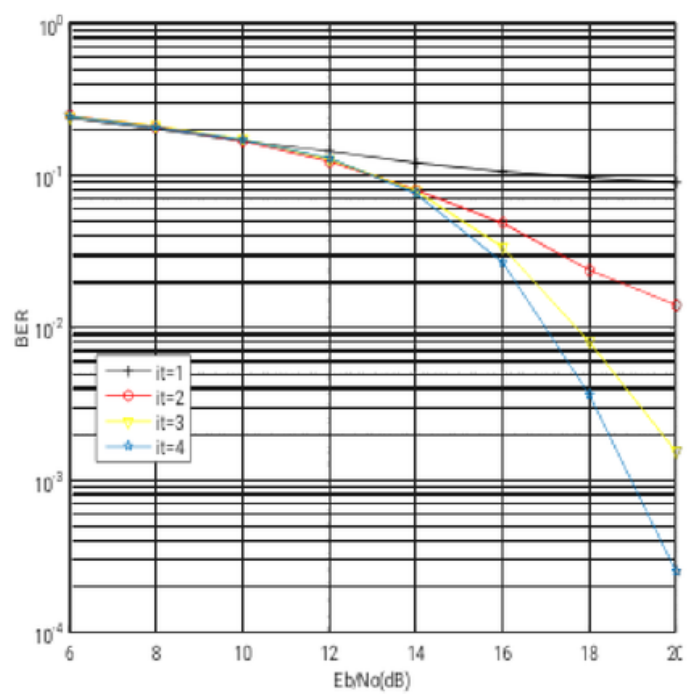

(b)

Fig. (8) Performance with ( $N=4, s p=4$ ) for (a) different block sizes $\left(N_{b}\right)$, and (b) different iterations ( it ).

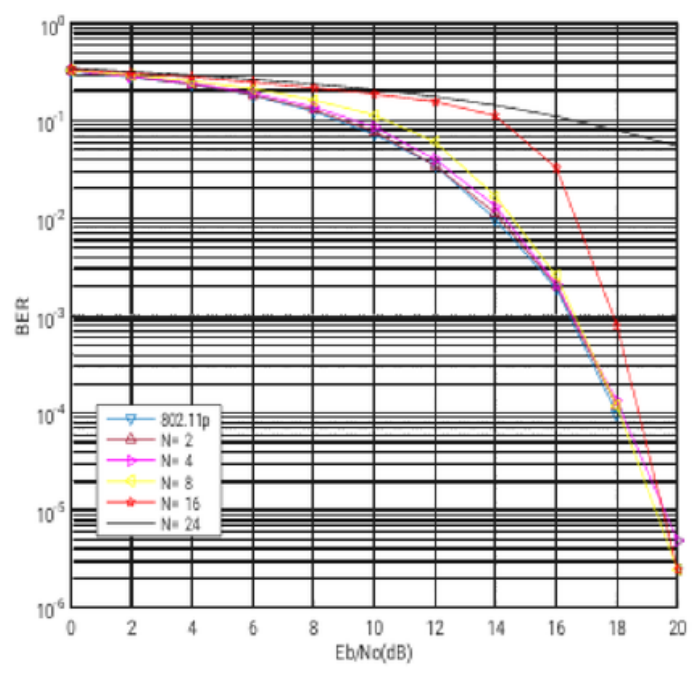

(a)

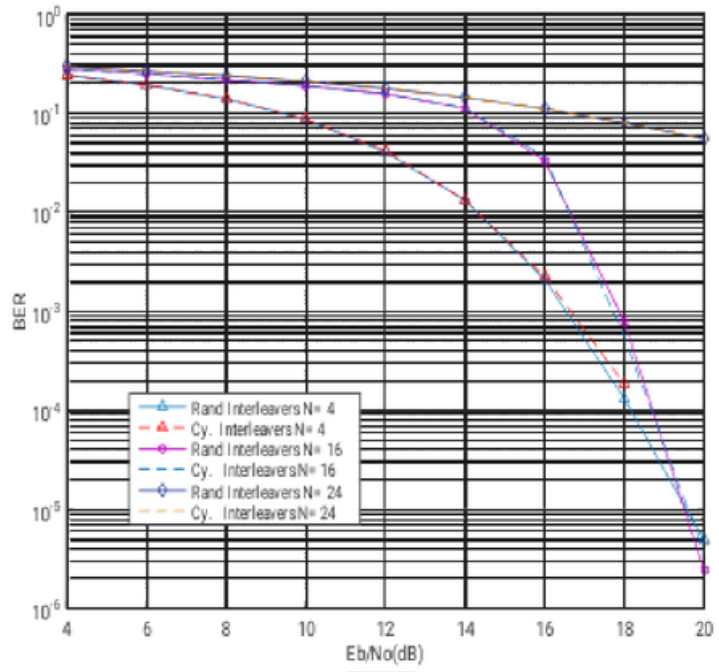

(b)

Fig. (9) Performance of the MPD-FDE with (it $=4, s p=16$ ) for (a) different number of nodes $(N)$, and (b) different interleavers. 


\subsection{Performance comparison with ${ }_{3}$ C schemes and channel coding}

Fig. 10(b) shows the performance $3 \mathrm{f}$ the coded and uncoded transmission over vehicular channels. Because of error detection and correction, 3 ER for coded transmission continues to fall sharply in comparison with uncoded transmission. Thus, the results with coding have lower errors compared with the simulation results without coding. In fig. 10(a), similar results are also shown for the IC schemes. In high $\frac{E_{b}}{N_{0}}$ regions, the BER is remarkably improved for both IC schemes. Both IC schemes show significant gain of the turbo detection. Further, SIC algorithm outperforms the PIC, which takes less time and complexity compared to the SIC algorithm.

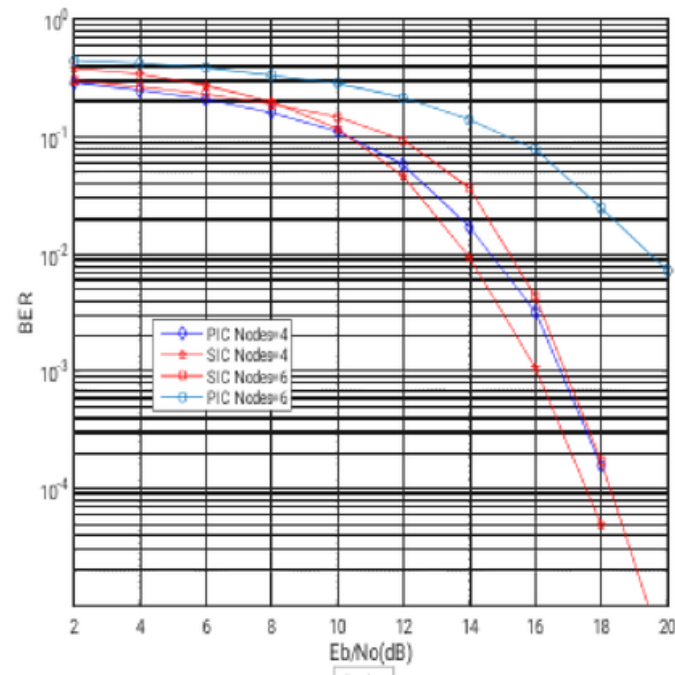

(a)

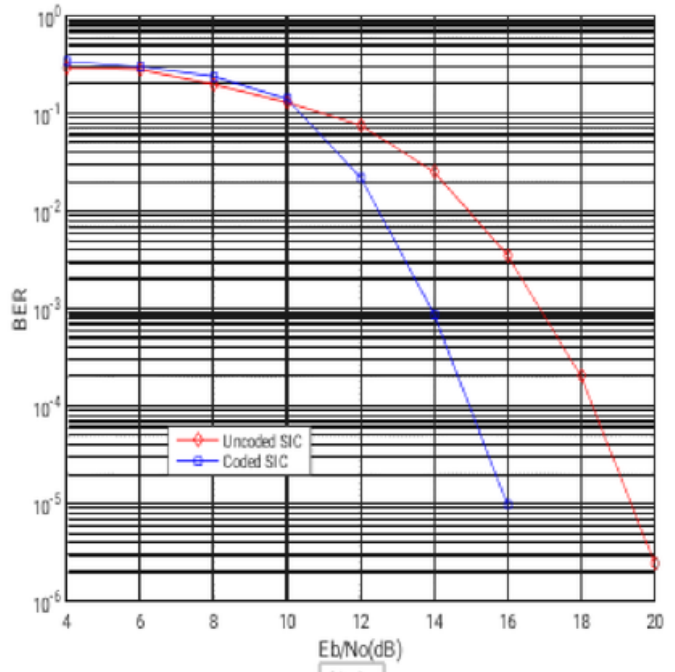

(b)

Fig. (10) Performance comparison of the MPD-FDE with (a) different IC schemes (it = 6, $s p=8$ ), and (b) uncoded \& coded transmission $(N=6, i t=3, s p=4)$.

\section{Conclusion}

In this paper, a $2 \mathrm{MAC}$ protocol in cooperating with PHY layer are proposed to satisfy the requirements of Ad hoc networks, with an emphasis on MPD-FDE as a flexible means for IC. The focus was on the scenario of uplink communication between multi nodes and RSU. The numerical results indicated that the performance of MPD-FDE with SIC scheme outperforms the PIC, but with much time delay. Further, the proposed detector with cyclically interleavers eventually obtains almost the same performance as with random interleavers with less memory resources. The presented work is part of a larger project and several aspects, such as multi hop scenarios, and channel estimation, need more investigation towards higher spectral efficiencies.

\section{References}

[1] S. Carlos, Successive Interference Cancellation in Vehicular Networks to Relieve the Negative Impact of the Hidden Node Problem, Master thesis in Electrical Engineering and Computer, CMSC Pereira, Sep. 2011.

[2] F. Jiang, C. Moloney and C. Li, "A Preliminary Investigation of Multi-user Interference Cancellation Techniques at Roadside Unit in Vehicular Networks", in Proc. of the 10th Intern. conf. on mobile Ad hoc and sensor networks, Maui, 2014, pp.158-162.

[3] H. Hartenstein and K. Laberteaux, "A tutorial survey on vehicular ad hoc networks", IEEE Comm. Magazine, vol. 46, no. 6, pp. 164-171, 2008. 
[4] K. Kusume, R. Vilzmann, and A. Müller, "Medium Access in Spread-Spectrum Ad Hoc Networks with Multiuser Detection", J. on Advances in Signal Proc. 2009, Jan. 2009.

[5] K. Kusume, R. Vilzmann, A. Müller, C. Hartmann, G. Bauch, "A Multiuser Detection Perspective on Medium Access Control in Ad Hoc Networks", in Proc. of the IEEE Global Telecom. Conf. GLOBECOM, Nov. 2007, pp. 801- 806.

[6] 3G TS 25.213, 3rd generation partnership project; technical specification group radio access network; spreading and modulation (FDD), (release 4), Jun. 2002.

[7] L. Ping, L. Liu, K. Y. Wu, and W. K. Leung, "Interleave-division multiple-access", IEEE Trans. on Wireless Comm., vol. 5, no. 4, pp. 938-947, Apr. 2006.

[8] L. Tong, V. Naware, and P. V. subramaniam, "Signal processing in random access", IEEE Signal Proc. Magazine, vol. 21, no. 5, pp. 29-39, Sep. 2004.

[9] R. Vilzmann and C. Bettstetter, "A survey on MAC protocols for ad hoc networks with directional antennas", in Proc. 11th EUNICE, Jul. 2005.

[10] M. Zorzi, J. Zeidler, A. Anderson, B. Rao, J. Proakis, A. L. Swindlehurst, M. Jensen, and S. Krishnamurthy, "Cross-layer issues in MAC protocol design for MIMO ad hoc networks", IEEE Wireless Comm., vol. 13, no. 4, pp. 62-76, 2006

[11] G. Foschini, "Layered space-time architecture for wireless communication in a fading environment when using multi-element antennas", Bell Labs. Tech. Journal, vol. 1, no. 2, pp. 41-59, 1996.

[12] D. Brown, "Multistage parallel interference cancellation: convergence behavior and improved performance through limit cycle mitigation", IEEE Trans. on Signal Proce., vol. 53, no. 1, pp. 283-294, 2005.

[13] D. Halperin, Th. Anderson, and D. Wetherall. "Taking the Sting Out of Carrier Sense: Interference Cancellation for Wireless LANs". In Proc. of the 14th ACM intern. Conf. MobiCom '08, New York, 2008, pp. 339-350.

[14] D. Divsalar, M. Simon, and D. Raphaeli, "Improved parallel interference cancellation for CDMA," IEEE Trans. on Comm., vol. 46, pp. 258-268, 1998.

[15] D. Brown, "Multistage parallel interference cancellation: convergence behavior and improved performance through limit cycle mitigation," IEEE Trans. on Signal Proc., vol. 53, no. 1, pp. 283-294, 2005.

[16] R. G. Gallager, "A perspective on multiaccess channels", IEEE Tran. on Information Theory, vol. 31, no. 2, pp. 124-142, Mar. 1985.

[17] A. Duel-Hallen, J. Holtzman, and Z. Zvonar. "Multiuser detection for CDMA systems". Personal Comm., IEEE, vol. 2, no. 2, pp. 46-58, Apr. 1995.

[18] J. G. Andrews, S. Weber, and M. Haenggi, "Ad hoc networks: To spread or not to spread? [ad hoc and sensor networks]," IEEE Comm. Magazine, vol. 45, no. 12, pp. 84-91, Dec. 2007.

[19] A. Muqattash, M. Krunz, and W. E. Ryan, "Solving the near-far problem in CDMA-based ad hoc networks", Ad Hoc Networks, vol. 1, no. 4, pp. 435-453, 2003.

[20] K. Kusume and G. Bauch, "Some aspects of interleave division multiple access in ad hoc networks," in Proc. Int. Symp. on ITG, Apr. 2006.

[21] J. B. Lim, C. H. Choi, T. W. Yune, and Gi-Hong Im, "Iterative Multiuser Detection for Single carrier Modulation with Frequency-domain Equalization", IEEE Comm.. Letters, vol. 11, no. 6, pp. 471-73, Jun. 2007.

[22] T. W. Yune, C. H. Choi, and G. H. Im, "Single carrier frequency-domain equalization with transmit diversity over mobile multipath channel", IEICE Trans. Comm., vol. 89, no. 7, pp. 2050-2060, Jul. 2006.

[23] T. W. Yune, C H. Choi, G. H. Im, Y. C. Cheong, and Ki-Ho Kim, "SC-FDMA with iterative multiuser detection: improvements on power/spectral efficiency", IEEE Comm. Magazine, vol. 48, no. 3, pp. 164 171, Mar. 2010.

[24] M. H. Eiza, Q. Ni, Th. Owens and G. Min, "Investigation of routing reliability of vehicular ad hoc networks", EURASIP J. on Wireless Comm. and Networking, 2013.

[25] N. M. Alfahad, Salah A. Aliesawi, and F. S. Mubarek, "Enhancing AODV routing protocol based on 
direction and velocity for real-time urban scenario", J. of Theoretical and Applied Information Technology, 2018.

[26] F. S. Mubarek, Salah A. Aliesawi, Khattab M. Ali Alheeti, N. M. Alfahad, "Urban-AODV: an improved AODV protocol for vehicular ad-hoc networks in urban environment", Intern. J. of Engineering and Technology (UAE), 2018.

[27] H. Spijker, Improving information dissemination in sparse vehicular networks by adding satellite communication, Master Thesis in computer, University of Twente, May 2012.

[28] S. Shooshtary, Development of a MATLAB Simulation Environment for Vehicle-to-Vehicle and Infrastructure Communication Based on IEEE 802.11p, Master's Thesis in Telecom., Vienna university, Dec. 2008.

[29] A. K. Pandey, Simulation of Traffic Movement in Vanet using SUMO", Bach. thesis in Comp. Science \& Eng., Nattional Inst. of Tech. , Rourkela, May 2013.

[30] S. Rehman, T. Zia and L. Zheng, " A Border Base Directional Routing (BBDR) Protocol for VANETs", Proc. of the 7th IB2COM, Nov. 5-8, 2012.

[31] W. Liang, Z. Li, and R. Bie, "Vehicular Ad Hoc Networks: Architectures, Research Issues, Methodologies, Challenges, and Trends", Hindawi Publishing Corp. Intern. J. of Distributed Sensor Net., vol. 2015.

[32] H. Hartenstein and K. Laberteaux, VANET-Vehicular Applications and Inter-Networking Technologies, John Wiley \& Sons, 2010.

[33] V. Loscri, "MAC Schemes for Ad-Hoc Wireless Networks", in Proc. VTC-2007 Fall Vehicular Technology Conf., pp. 36-40, 2007.

[34] Benjamin Durakovic, "Design for Additive Manufacturing: Benefits, Trends and Challenges", Periodicals of Engineering and Natural Sciences Vol.6, No.2, pp. 179-191, (2018), ISSN 2303-4521.

[35] Benjamin Durakovic, et al, "Lean Manufacturing: Trends and Implementation Issues", Periodical of Engineering and Natural Sciences, Vol. 6, No. 1, pp. 130-143 (2018), ISSN: 2303-4521.

[36] Ramo Palalic, Benjamin Durakovic, "Does Transformational Leadership Matters in Gazelles and Mice: Evidence from Bosnia and Herzegovina?", International Journal of Entrepreneurship and Small Business, Vol. 34, No. 3, pp. 289-308 (2018), ISSN: 1476-1297. DOI: 10.1504/IJESB.2018.092744

[37] Benjamin Durakovic, "Design of Experiments Application, Concepts, Examples: State of the Art", Periodical of Engineering and Natural Sciences, Vol. 5, No. 3, pp. 421-439 (2017), ISSN: 2303-4521. 
Iterative Multipacket Detection with FDE based MAC Protocol in Vehicular Ad Hoc Networks

ORIGINALITY REPORT

$8 \%$

SIMILARITY INDEX
$1 \%$

INTERNET SOURCES
$6 \%$

PUBLICATIONS
$6 \%$

STUDENT PAPERS

PRIMARY SOURCES

1 Submitted to Asia Pacific University College of Technology and Innovation (UCTI)

Student Paper

2 pdfs.semanticscholar.org

Internet Source

3 Submitted to TechKnowledge

Student Paper

4 Wenshuang Liang, Zhuorong Li, Hongyang

Zhang, Shenling Wang, Rongfang Bie.

"Vehicular Ad Hoc Networks: Architectures,

Research Issues, Methodologies, Challenges,

and Trends", International Journal of Distributed

Sensor Networks, 2015

Publication

5

Submitted to Sheffield Hallam University

Student Paper

Multi-Carrier Spread Spectrum 2007, 2007.

Publication 
On 